\title{
A Study of Uniform Harmonic $\chi$-Convex Functions with respect to Hermite-Hadamard's Inequality and Its Caputo-Fabrizio Fractional Analogue and Applications
}

\author{
Miguel Vivas-Cortez $\left(\mathbb{D},{ }^{1}\right.$ Muhammad Uzair Awan $\left(\mathbb{D},{ }^{2}\right.$ Muhammad Zakria Javed, ${ }^{2}$ \\ Muhammad Aslam Noor $\left(\mathbb{D},{ }^{3}\right.$ and Khalida Inayat Noor $\mathbb{D}^{3}$ \\ ${ }^{1}$ Escuela de Ciencias Físicas y Matemáticas, Facultad de Ciencias Exactas y Naturales, Pontificia Universidad Católica del Ecuador, \\ Av. 12 de Octubre 1076, Apartado, Quito 17-01-2184, Ecuador \\ ${ }^{2}$ Department of Mathematics, Government College University, Faisalabad, Pakistan \\ ${ }^{3}$ Department of Mathematics, COMSATS University Islamabad, Islamabad, Pakistan
}

Correspondence should be addressed to Muhammad Uzair Awan; awan.uzair@gmail.com

Received 21 August 2021; Revised 11 October 2021; Accepted 11 November 2021; Published 3 December 2021

Academic Editor: John R. Akeroyd

Copyright (c) 2021 Miguel Vivas-Cortez et al. This is an open access article distributed under the Creative Commons Attribution License, which permits unrestricted use, distribution, and reproduction in any medium, provided the original work is properly cited.

In this paper, we introduce the notion of uniform harmonic $\chi$-convex functions. We show that this class relates several other unrelated classes of uniform harmonic convex functions. We derive a new version of Hermite-Hadamard's inequality and its fractional analogue. We also derive a new fractional integral identity using Caputo-Fabrizio fractional integrals. Utilizing this integral identity as an auxiliary result, we obtain new fractional Dragomir-Agarwal type of inequalities involving differentiable uniform harmonic $\chi$-convex functions. We discuss numerous new special cases which show that our results are quite unifying. Finally, in order to show the significance of the main results, we discuss some applications to means of positive real numbers.

\section{Preliminaries}

In recent years, the classical concepts of convexity have been extended and generalized in different directions using novel and innovative ideas. It has also been observed that these new extensions and generalizations enjoy some nice properties which the classical concepts of convexity have. Everyone is familiar with the fact that convexity has a close relationship with the theory of inequalities. The classical concepts of convexity have played a significant role in the development of the theory of inequalities. Many famously known results in the theory of inequalities can easily be obtained using the convexity property of the functions. Thus, this becomes an interesting problem for research, of obtaining new versions of classical inequalities using new generalizations of convexity. Interested readers may find some useful details regarding convexity, its generalizations, and associ- ated results in [1]. In 2014, Işcan [2] introduced and investigated the notion of harmonic convex functions. He defined the class of harmonic convex functions as follows.

Definition 1. A function $Y: H \subset(0,+\infty) \longrightarrow \mathbb{R}$ is said to be harmonically convex, if

$$
Y\left(\frac{x y}{i x+(1-i) y}\right) \leq i Y(y)+(1-i) Y(x)
$$

holds for all $x, y \in H$ and $i \in[0,1]$.

He also obtained a new variant of Hermite-Hadamard's inequality pertaining to harmonic convex functions as follows. 
Theorem 2. Let $Y: I=\left[b_{1}, b_{2}\right] \subset(0, \infty) \longrightarrow \mathbb{R}$ be a harmonically convex function, and then

$$
Y\left(\frac{2 b_{1} b_{2}}{b_{1}+b_{2}}\right) \leq \frac{b_{1} b_{2}}{b_{2}-b_{1}} \int_{b_{1}}^{b_{2}} Y(x) d x \leq \frac{Y\left(b_{1}\right)+Y\left(b_{2}\right)}{2} .
$$

Having inspiration from the research work of Sarikaya et al. [3], Isscan and $\mathrm{Wu}$ [4] obtained the fractional version of Hermite-Hadamard's inequality, essentially utilizing the concept of harmonic convex functions. For some recent investigations on harmonic convex functions, see $[5,6]$.

In [7], the class of uniformly convex functions was defined as follows.

Definition 3. Let $K \subset \mathbb{R}$ be a convex set. A function $Y: K$ $\longrightarrow \mathbb{R}$ is said to be uniformly convex with modulus $\psi:[0$, $\infty] \longrightarrow[0, \infty]$ if $\psi$ is increasing, $\psi$ vanishes only at 0 , and

$$
\begin{array}{r}
Y((1-i) x+i y)+i(1-i) \psi(|y-x|) \leq(1-i) Y(x)+i Y(y), \\
\forall x, y \in K, i \in[0,1] .
\end{array}
$$

The main motivation of this paper is to introduce the class of uniform harmonic $\chi$-convex functions. We show that this class contains some other new classes of uniform harmonic convex functions. Using the class of uniform harmonic $\chi$-convex functions, we also obtain some new versions of Hermite-Hadamard's inequality, essentially utilizing the concepts from both ordinary calculus and fractional calculus. In order to show the significance of the main results, we present some applications to special means of real numbers.

Before we proceed further, let us recall some previously known concepts from fractional calculus. The CaputoFabrizio fractional derivative and fractional integrals are defined, respectively, as follows.

Let $Y \in H^{1}\left(b_{1}, b_{2}\right), b_{1}<b_{2}, \alpha \in[0,1]$, and then the definition of the left fractional derivative is defined as follows:

$$
\left({ }_{b_{1}}^{C F C} D^{\alpha}\right)(i)=\frac{B(\alpha)}{1-\alpha} \int_{b_{1}}^{i} Y^{\prime}(x) \exp \frac{-\alpha(i-x)^{\alpha}}{1-\alpha} d x
$$

and the associated fractional integral is defined as follows:

$$
\left({ }_{b_{1}}^{C F} I^{\alpha}\right)(i)=\frac{1-\alpha}{B(\alpha)} Y(i)+\frac{\alpha}{B(\alpha)} \int_{b_{1}}^{i} Y(x) d x
$$

The right Caputo-Fabrizio fractional derivative is defined as follows:

$$
\left({ }^{C F C} D_{b_{2}}^{\alpha}\right)(i)=\frac{-B(\alpha)}{1-\alpha} \int_{i}^{b_{2}} f^{\prime}(x) \exp \frac{-\alpha(x-i)^{\alpha}}{1-\alpha} d x,
$$

and the associated right fractional integral is defined as follows:

$$
\left({ }^{C F} I_{b_{2}}^{\alpha}\right)(i)=\frac{1-\alpha}{B(\alpha)} Y(i)+\frac{\alpha}{B(\alpha)} \int_{i}^{b_{2}} f(x) d x,
$$

where $B(\alpha)>0$ is the normalization function satisfying $B$ $(0)=B(1)=1$. For more details, see $[8,9]$.

Our calculations involve beta and hypergeometric functions. For the sake of completeness, let us recall these classical concepts. The beta and hypergeometric functions are defined as

$$
\mathscr{B}(x, y)=\int_{0}^{1} v^{x-1}(1-v)^{y-1} d v,
$$

or

$$
\begin{aligned}
& \mathscr{B}(x, y)=\frac{\Gamma(x) \Gamma(y)}{\Gamma(x+y)}, \\
&{ }_{2} \mathscr{F}_{1}(x, y ; c ; z)=\frac{1}{\mathscr{B}(y, c-y)} \int_{0}^{1} v^{y-1}(1-v)^{c-y-1}(1-z v)^{-x} d v, \\
& c>y>0,|z|<1 .
\end{aligned}
$$

\section{Main Results}

In this section, we discuss our main results.

2.1. Uniform Harmonic $\chi$-Convex Functions. We now introduce the class of uniform harmonic $\chi$-convex functions.

Definition 4. Let $\chi:(0,1) \longrightarrow \mathbb{R}$ be a real function. A function $Y:\left[b_{1}, b_{2}\right] \subset(0, \infty) \longrightarrow \mathbb{R}$ is said to be a uniform harmonic $\chi$-convex function, if

$$
\begin{aligned}
Y\left(\frac{b_{1} b_{2}}{(1-i) b_{1}+i b_{2}}\right) \leq & \chi(i) Y\left(b_{1}\right)+\chi(1-i) Y\left(b_{2}\right) \\
& -(1-i) \chi(i) \psi\left(\left|\frac{1}{b_{1}}-\frac{1}{b_{2}}\right|\right) .
\end{aligned}
$$

Some special cases of Definition 4 are enlisted as follows:

(i) If we take $\chi(i)=i$, then we obtain the class of uniform harmonic convex function, which is defined as

(ii) If we take $\chi(i)=i^{s}$, then we obtain the class of uniform harmonic $s$-convex function, which is defined as

(iii) If we take $\chi(i)=i^{-s}$, then we obtain the class of the Godunova-Levin-Dragomir (GLD) type of uniform harmonic $s$-convex function, which is defined as

(iv) If we take $\chi(i)=i$ and $\psi(i)=\delta i^{2}$, then we obtain the class of strongly uniform harmonic convex function, which is defined as 
(v) If we take $\chi(i)=i$ and $\psi(i)=-\delta|i|$, then we obtain the class of approximate harmonic convex function, which is defined as

(vi) If we take $\chi(i)=i$ and $\psi(i)=-\delta|i|^{p}$, then we obtain the class of approximate harmonic convex function of order $p>0$, which is defined as

Definition 5. A function $Y:\left[b_{1}, b_{2}\right] \subset(0, \infty) \longrightarrow \mathbb{R}$ is said to be a uniform harmonic convex function, if

$Y\left(\frac{b_{1} b_{2}}{(1-i) b_{1}+i b_{2}}\right) \leq i Y\left(b_{1}\right)+(1-i) Y\left(b_{2}\right)-(1-i) i \psi\left(\left|\frac{1}{b_{1}}-\frac{1}{b_{2}}\right|\right)$.

Definition 6. A function $Y:\left[b_{1}, b_{2}\right] \subset(0, \infty) \longrightarrow \mathbb{R}$ is said to be a uniform harmonic $s$-convex function, if

$Y\left(\frac{b_{1} b_{2}}{(1-i) b_{1}+i b_{2}}\right) \leq i^{s} f\left(b_{1}\right)+(1-i)^{s} f\left(b_{2}\right)-(1-i) i^{s} \psi\left(\left|\frac{1}{b_{1}}-\frac{1}{b_{2}}\right|\right)$.

Definition 7. A function $Y:\left[b_{1}, b_{2}\right] \subset(0, \infty) \longrightarrow \mathbb{R}$ is said to be a Godunova-Levin-Dragomir (GLD) type of uniform harmonic $s$-convex functions, if

$Y\left(\frac{b_{1} b_{2}}{(1-i) b_{1}+i b_{2}}\right) \leq i^{-s} Y\left(b_{1}\right)+(1-i)^{-s} Y\left(b_{2}\right)-(1-i) i^{-s} \psi\left(\left|\frac{1}{b_{1}}-\frac{1}{b_{2}}\right|\right)$.

Definition 8. A function $Y:\left[b_{1}, b_{2}\right] \subset(0, \infty) \longrightarrow \mathbb{R}$ is said to be a strongly uniform harmonic convex function, if

$Y\left(\frac{b_{1} b_{2}}{(1-i) b_{1}+i b_{2}}\right) \leq i Y\left(b_{1}\right)+(1-i) Y\left(b_{2}\right)-\delta(1-i) i\left|\frac{1}{b_{1}}-\frac{1}{b_{2}}\right|^{2}$.

Definition 9. A function $Y:\left[b_{1}, b_{2}\right] \subset(0, \infty) \longrightarrow \mathbb{R}$ is said to be an approximate uniform harmonic convex function, if

$Y\left(\frac{b_{1} b_{2}}{(1-i) b_{1}+i b_{2}}\right) \leq i Y\left(b_{1}\right)+(1-i) Y\left(b_{2}\right)+\delta(1-i) i\left|\frac{1}{b_{1}}-\frac{1}{b_{2}}\right|$.

Definition 10. A function $Y:\left[b_{1}, b_{2}\right] \subset(0, \infty) \longrightarrow \mathbb{R}$ is said to be an approximate uniform harmonic convex function of order $p>0$, if

$Y\left(\frac{b_{1} b_{2}}{(1-i) b_{1}+i b_{2}}\right) \leq i Y\left(b_{1}\right)+(1-i) Y\left(b_{2}\right)+\delta(1-i) i\left|\frac{1}{b_{1}}-\frac{1}{b_{2}}\right|^{p}$.

2.2. A New Hermite-Hadamard's Inequality. We now derive a new variant of Hermite-Hadamard's inequality using the class of uniform harmonic $\chi$-convex functions. We also discuss some new special cases of this result.
Theorem 11. Let $Y:\left[b_{1}, b_{2}\right] \subset(0, \infty) \longrightarrow \mathbb{R}$ be a uniform harmonic $\chi$-convex function, and then

$$
\begin{aligned}
& \frac{1}{\chi(1 / 2)} Y\left(\frac{2 b_{1} b_{2}}{b_{1}+b_{2}}\right)+\int_{\left(b_{1}-b_{2}\right) / b_{1} b_{2}}^{\left(b_{2}-b_{1}\right) / b_{1} b_{2}} \frac{b_{1} b_{2}}{4\left(b_{2}-b_{1}\right)} \psi(i) d i \\
& \leq \frac{2 b_{1} b_{2}}{b_{2}-b_{1}} \int_{1 / b_{2}}^{1 / b_{1}}(Y \circ \Lambda)(i) d i \\
& \leq\left[Y\left(b_{1}\right)+Y\left(b_{2}\right)\right] \int_{0}^{1}[\chi(i)+\chi(1-i)] d i \\
& \quad-2 \int_{0}^{1}(1-i) \chi(i) \psi\left(\left|\frac{1}{b_{1}}-\frac{1}{b_{2}}\right|\right) d i,
\end{aligned}
$$

where $\Lambda(i)=1 / i, \forall i \in\left[b_{1}, b_{2}\right]$.

Proof. Since $Y$ is a uniform harmonic $\chi$-convex function, then for $i=1 / 2$, we have

$$
Y\left(\frac{2 x y}{x+y}\right) \leq \chi\left(\frac{1}{2}\right)\left[Y(x)+Y(y)-\frac{1}{2} \psi\left(\left|\frac{1}{x}-\frac{1}{y}\right|\right)\right] .
$$

Using the change of variable technique, we have

$$
\begin{aligned}
\frac{1}{\chi(1 / 2)} Y\left(\frac{2 b_{1} b_{2}}{b_{1}+b_{2}}\right) \leq & \int_{0}^{1} Y\left(\frac{b_{1} b_{2}}{(1-i) b_{1}+i b_{2}}\right) d i \\
& +\int_{0}^{1} Y\left(\frac{b_{1} b_{2}}{i b_{1}+(1-i) b_{2}}\right) d i \\
& -\int_{0}^{1} \frac{1}{2} \psi\left(\left|\frac{\left(b_{2}-b_{1}\right)(2 i-1)}{b_{1} b_{2}}\right|\right) d i
\end{aligned}
$$

After simplifying, we obtain

$$
\begin{aligned}
& \frac{1}{\chi(1 / 2)} Y\left(\frac{2 b_{1} b_{2}}{b_{1}+b_{2}}\right)+\int_{\left(b_{1}-b_{2}\right) / b_{1} b_{2}}^{\left(b_{2}-b_{1}\right) / b_{1} b_{2}} \frac{b_{1} b_{2}}{4\left(b_{2}-b_{1}\right)} \psi(i) d i \\
& \quad \leq \frac{2 b_{1} b_{2}}{b_{2}-b_{1}} \int_{1 / b_{2}}^{1 / b_{1}}(Y \circ \Lambda)(i) d i .
\end{aligned}
$$

Now, we prove our second inequality using the notion of uniform harmonic $\chi$ convexity.

$$
Y\left(\frac{b_{1} b_{2}}{(1-i) b_{1}+i b_{2}}\right) \leq \chi(i) Y\left(b_{1}\right)+\chi(1-i) Y\left(b_{2}\right)-(1-i) \chi(i) \psi\left(\left|\frac{1}{b_{1}}-\frac{1}{b_{2}}\right|\right)
$$

$$
Y\left(\frac{b_{1} b_{2}}{i b_{1}+(1-i) b_{2}}\right) \leq \chi(1-i) Y\left(b_{1}\right)+\chi(i) Y\left(b_{2}\right)-(1-i) \chi(i) \psi\left(\left|\frac{1}{b_{1}}-\frac{1}{b_{2}}\right|\right) .
$$


Adding (21) and (22), we have

$$
\begin{aligned}
& Y\left(\frac{b_{1} b_{2}}{(1-i) b_{1}+i b_{2}}\right)+Y\left(\frac{b_{1} b_{2}}{i b_{1}+(1-i) b_{2}}\right) \\
& \quad \leq\left[Y\left(b_{1}\right)+Y\left(b_{2}\right)\right][\chi(i)+\chi(1-i)]-2(1-i) \chi(i) \psi\left(\left|\frac{1}{b_{1}}-\frac{1}{b_{2}}\right|\right) .
\end{aligned}
$$

Integrating both sides with respect to $i$ on $[0,1]$, then we have

$$
\begin{aligned}
\frac{2 b_{1} b_{2}}{b_{2}-b_{1}} \int_{1 / b_{2}}^{1 / b_{1}}(Y \circ \Lambda)(i) d i \leq & {\left[Y\left(b_{1}\right)+Y\left(b_{2}\right)\right] \int_{0}^{1}[\chi(i)+\chi(1-i)] d i } \\
& -2 \int_{0}^{1}(1-i) \chi(i) \psi\left(\left|\frac{1}{b_{1}}-\frac{1}{b_{2}}\right|\right) d i .
\end{aligned}
$$

This completes the proof.

We now discuss some special cases of Theorem 11:

(i) If we take $\chi(i)=i$ in Theorem 11 , then we have the result for uniform harmonic convex functions

(ii) If we take $\chi(i)=i^{s}$ in Theorem 11 , then we have the result for uniform harmonic $s$-convex functions

(iii) If we take $\chi(i)=i^{-s}$ in Theorem 11, then we have the result for the GLD type of uniform harmonic $s$ -convex functions

Corollary 12. Under the assumptions of Theorem 11, if $Y$ is a uniform harmonic convex function, then

$$
\begin{aligned}
& Y\left(\frac{2 b_{1} b_{2}}{b_{1}+b_{2}}\right)+\int_{\left(b_{1}-b_{2}\right) / b_{1} b_{2}}^{\left(b_{2}-b_{1}\right) / b_{1} b_{2}} \frac{b_{1} b_{2}}{8\left(b_{2}-b_{1}\right)} \psi(i) d i \\
& \leq \frac{b_{1} b_{2}}{b_{2}-b_{1}} \int_{1 / b_{2}}^{1 / b_{1}}(Y \circ \Lambda)(i) d i \leq \frac{Y\left(b_{1}\right)+Y\left(b_{2}\right)}{2} \\
& \quad-\frac{1}{6} \psi\left(\left|\frac{1}{b_{1}}-\frac{1}{b_{2}}\right|\right) d i .
\end{aligned}
$$

Corollary 13. Under the assumptions of Theorem 11, if $Y$ is a uniform harmonic s-convex function, then

$$
\begin{aligned}
& 2^{s-1} Y\left(\frac{2 b_{1} b_{2}}{b_{1}+b_{2}}\right)+\int_{\left(b_{1}-b_{2}\right) / b_{1} b_{2}}^{\left(b_{2}-b_{1}\right) / b_{1} b_{2}} \frac{b_{1} b_{2}}{8\left(b_{2}-b_{1}\right)} \psi(i) d i \\
& \quad \leq \frac{b_{1} b_{2}}{b_{2}-b_{1}} \int_{1 / b_{2}}^{1 / b_{1}}(Y \circ \Lambda)(i) d i \\
& \quad \leq \frac{Y\left(b_{1}\right)+Y\left(b_{2}\right)}{s+1}-\frac{1}{(s+1)(s+2)} \psi\left(\left|\frac{1}{b_{1}}-\frac{1}{b_{2}}\right|\right) d i .
\end{aligned}
$$

Corollary 14. Under the assumptions of Theorem 11, if $Y$ is a GLD type of uniform harmonic s-convex functions, then

$$
\begin{aligned}
& 2^{1-s} Y\left(\frac{2 b_{1} b_{2}}{b_{1}+b_{2}}\right)+\int_{\left(b_{1}-b_{2}\right) / b_{1} b_{2}}^{\left(b_{2}-b_{1}\right) / b_{1} b_{2}} \frac{b_{1} b_{2}}{8\left(b_{2}-b_{1}\right)} \psi(i) d i \\
& \quad \leq \frac{b_{1} b_{2}}{b_{2}-b_{1}} \int_{1 / b_{2}}^{1 / b_{1}}(Y \circ \Lambda)(i) d i \\
& \quad \leq \frac{Y\left(b_{1}\right)+Y\left(b_{2}\right)}{1-s}-\frac{1}{(1-s)(2-s)} \psi\left(\left|\frac{1}{b_{1}}-\frac{1}{b_{2}}\right|\right) d i
\end{aligned}
$$

2.3. Fractional Hermite-Hadamard's Inequality Using Caputo-Fabrizio Fractional Integrals. We now derive a fractional version of Theorem 11 by using Caputo-Fabrizio fractional integrals.

Theorem 15. Let $Y:\left[b_{1}, b_{2}\right] \longrightarrow \mathbb{R}$ be a uniform harmonic $\chi$-convex function, and then

$$
\begin{aligned}
& \frac{1}{\chi(1 / 2)} Y\left(\frac{2 b_{1} b_{2}}{b_{1}+b_{2}}\right)+\frac{b_{1} b_{2}}{4\left(b_{2}-b_{1}\right)} \int_{\left(b_{1}-b_{2}\right) / b_{1} b_{2}}^{\left(b_{2}-b_{1}\right) / b_{1} b_{2}} \psi(|i|) d i \\
& \quad+\frac{4 b_{1} b_{2}(1-\alpha)}{\alpha\left(b_{2}-b_{1}\right)}(Y \circ \Lambda)(k) \\
& \leq \frac{2 b_{1} b_{2} B(\alpha)}{\alpha\left(b_{2}-b_{1}\right)}\left[\left({ }_{1 / b_{2}}^{C F} I^{\alpha} Y \circ \Lambda\right)(k)+\left({ }_{1 / b_{1}}^{C F} I^{\alpha} Y \circ \Lambda\right)(k)\right] \\
& \leq \frac{4 b_{1} b_{2}(1-\alpha)}{\alpha\left(b_{2}-b_{1}\right)}(Y \circ \Lambda)(k)+\left[Y\left(b_{1}\right)+Y\left(b_{2}\right)\right] \\
& \quad \times \int_{0}^{1}[\chi(i)+\chi(1-i)] d i-2 \int_{0}^{1}(1-i) \chi(i) \psi\left(\left|\frac{1}{b_{1}}-\frac{1}{b_{2}}\right|\right) d i
\end{aligned}
$$

Proof. Since $Y$ is a uniform harmonic $\chi$-convex function, then

$$
\begin{aligned}
& \frac{1}{\chi(1 / 2)} Y\left(\frac{2 b_{1} b_{2}}{b_{1}+b_{2}}\right)+\int_{\left(b_{1}-b_{2}\right) / b_{1} b_{2}}^{\left(b_{2}-b_{1}\right) / b_{1} b_{2}} \frac{b_{1} b_{2}}{4\left(b_{2}-b_{1}\right)} \psi(i) d i \\
& \quad \leq \frac{2 b_{1} b_{2}}{b_{2}-b_{1}} \int_{1 / b_{2}}^{1 / b_{1}}(Y \circ \Lambda)(i) d i .
\end{aligned}
$$

The above inequality can be written as

$$
\begin{aligned}
& \frac{1}{\chi(1 / 2)} Y\left(\frac{2 b_{1} b_{2}}{b_{1}+b_{2}}\right)+\int_{\left(b_{1}-b_{2}\right) / b_{1} b_{2}}^{\left(b_{2}-b_{1}\right) / b_{1} b_{2}} \frac{b_{1} b_{2}}{4\left(b_{2}-b_{1}\right)} \psi(i) d i \\
& \quad \leq \frac{2 b_{1} b_{2}}{b_{2}-b_{1}}\left[\int_{1 / b_{2}}^{k}(Y \circ \Lambda)(i) d i+\int_{k}^{1 / b_{1}}(Y \circ \Lambda)(i) d i\right] .
\end{aligned}
$$


Multiplying both sides by $\alpha\left(b_{2}-b_{1}\right) / 2 b_{1} b_{2} B(\alpha)$, adding $(2(1-\alpha) / B(\alpha))(Y \circ \Lambda)(k)$, and using Caputo-Fabrizio fractional integrals, we have

$$
\begin{aligned}
& \frac{\alpha\left(b_{2}-b_{1}\right)}{2 b_{1} b_{2} B(\alpha) \chi(1 / 2)} Y\left(\frac{2 b_{1} b_{2}}{b_{1}+b_{2}}\right)+\frac{\alpha}{8 B(\alpha)} \int_{\left(b_{1}-b_{2}\right) / b_{1} b_{2}}^{\left(b_{2}-b_{1}\right) / b_{1} b_{2}} \psi(|i|) d i \\
& \quad+\frac{2(1-\alpha)}{B(\alpha)}(Y \circ \Lambda)(k) \\
& \leq \frac{2(1-\alpha)}{B(\alpha)}(Y \circ \Lambda)(k)+\frac{\alpha}{B(\alpha)}\left[\int_{1 / b_{2}}^{k}(Y \circ \Lambda)(i) d i+\int_{k}^{1 / b_{1}}(Y \circ \Lambda)(i) d i\right] .
\end{aligned}
$$

This implies

$$
\begin{aligned}
& \frac{\alpha\left(b_{2}-b_{1}\right)}{2 b_{1} b_{2} B(\alpha) \chi(1 / 2)} Y\left(\frac{2 b_{1} b_{2}}{b_{1}+b_{2}}\right)+\frac{\alpha}{8 B(\alpha)} \int_{\left(b_{1}-b_{2}\right) / b_{1} b_{2}}^{\left(b_{2}-b_{1}\right) / b_{1} b_{2}} \psi(|i|) d i \\
& \quad+\frac{2(1-\alpha)}{B(\alpha)}(Y \circ \Lambda)(k) \\
& \leq\left[\left(\begin{array}{l}
C F \\
1 / b_{2}
\end{array} I^{\alpha} Y \circ \Lambda\right)(k)+\left(\begin{array}{l}
C F \\
1 / b_{1}
\end{array} I^{\alpha} Y \circ \Lambda\right)(k)\right] .
\end{aligned}
$$

Thus, we have

$$
\begin{aligned}
& \frac{1}{\chi(1 / 2)} Y\left(\frac{2 b_{1} b_{2}}{b_{1}+b_{2}}\right)+\frac{b_{1} b_{2}}{4\left(b_{2}-b_{1}\right)} \int_{\left(b_{1}-b_{2}\right) / b_{1} b_{2}}^{\left(b_{2}-b_{1}\right) / b_{1} b_{2}} \psi(|i|) d i \\
& \quad+\frac{4 b_{1} b_{2}(1-\alpha)}{\alpha\left(b_{2}-b_{1}\right)}(Y \circ \Lambda)(k) \\
& \leq \frac{2 b_{1} b_{2} B(\alpha)}{\alpha\left(b_{2}-b_{1}\right)}\left[\left(\begin{array}{ll}
C F \\
1 / b_{2}
\end{array} I^{\alpha} Y \circ \Lambda\right)(k)+\left({ }_{1 / b_{1}}^{C F} I^{\alpha} Y \circ \Lambda\right)(k)\right] .
\end{aligned}
$$

Now, we compute our second inequality. Since $Y$ is a uniform harmonic $\chi$-convex function, we have

$$
\begin{aligned}
\frac{2 b_{1} b_{2}}{b_{2}-b_{1}} \int_{1 / b_{2}}^{1 / b_{1}}(Y \circ \Lambda)(i) d i \leq & {\left[Y\left(b_{1}\right)+Y\left(b_{2}\right)\right] \int_{0}^{1}[\chi(i)+\chi(1-i)] d i } \\
& -2 \int_{0}^{1}(1-i) \chi(i) \psi\left(\left|\frac{1}{b_{1}}-\frac{1}{b_{2}}\right|\right) d i .
\end{aligned}
$$

Multiplying both sides by $\alpha\left(b_{2}-b_{1}\right) / 2 b_{1} b_{2} B(\alpha)$, adding $(2(1-\alpha) / B(\alpha))(Y \circ \Lambda)(k)$, and using Caputo-Fabrizio fractional integrals, we have

$$
\begin{aligned}
& \frac{2(1-\alpha)}{B(\alpha)}(Y \circ \Lambda)(k)+\frac{\alpha}{B(\alpha)}\left[\int_{1 / b_{2}}^{k}(Y \circ \Lambda)(i) d i+\int_{k}^{1 / b_{1}}(Y \circ \Lambda)(i) d i\right] \\
& \leq \frac{2(1-\alpha)}{B(\alpha)}(Y \circ \Lambda)(k)+\frac{\alpha\left(b_{2}-b_{1}\right)}{2 b_{1} b_{2} B(\alpha)}\left[Y\left(b_{1}\right)+Y\left(b_{2}\right)\right] \\
& \quad \times \int_{0}^{1}[\chi(i)+\chi(1-i)] d i-\frac{\alpha\left(b_{2}-b_{1}\right)}{b_{1} b_{2} B(\alpha)} \int_{0}^{1}(1-i) \chi(i) \psi\left(\left|\frac{1}{b_{1}}-\frac{1}{b_{2}}\right|\right) d i .
\end{aligned}
$$

This implies

$$
\begin{aligned}
& \frac{2 b_{1} b_{2} B(\alpha)}{\alpha\left(b_{2}-b_{1}\right)}\left[\left(\begin{array}{l}
C F \\
1 / b_{2}
\end{array} I^{\alpha} Y \circ \Lambda\right)(k)+\left(\begin{array}{l}
C F \\
1 / b_{1}
\end{array} I^{\alpha} Y \circ \Lambda\right)(k)\right] \\
& \leq \frac{4 b_{1} b_{2}(1-\alpha)}{\alpha\left(b_{2}-b_{1}\right)}(Y \circ \Lambda)(k)+\left[Y\left(b_{1}\right)+Y\left(b_{2}\right)\right] \\
& \quad \times \int_{0}^{1}[\chi(i)+\chi(1-i)] d i-2 \int_{0}^{1}(1-i) \chi(i) \psi\left(\left|\frac{1}{b_{1}}-\frac{1}{b_{2}}\right|\right) d i .
\end{aligned}
$$

This completes the proof.

Now, we discuss some special cases of Theorem 15:

(i) If we take $\chi(i)=i$ in Theorem 15, then we have the result for uniform harmonic convex functions

(ii) If we take $\chi(i)=i$ and $\psi(i)=(\delta / 2) i^{2}$ in Theorem 15, then we have the result for strongly harmonic convex functions

(iii) If we take $\chi(i)=i^{s}$ in Theorem 15, then we have the result for uniform harmonic $s$-convex functions

(iv) If we take $\chi(i)=i^{s}$ and $\psi(i)=(\delta / 2) i^{2}$ in Theorem 15 , then we have the result for strongly harmonic $s$-convex functions

(v) If we take $\chi(i)=i^{-s}$ in Theorem 15, then we have the result for the GLD type of harmonic s-convex functions

Corollary 16. Under the assumptions of Theorem 15, if $Y$ is a uniform harmonic convex function, then

$$
\begin{aligned}
& Y\left(\frac{2 b_{1} b_{2}}{b_{1}+b_{2}}\right)+\frac{b_{1} b_{2}}{8\left(b_{2}-b_{1}\right)} \int_{\left(b_{1}-b_{2}\right) / b_{1} b_{2}}^{\left(b_{2}-b_{1}\right) / b_{1} b_{2}} \psi(|i|) d i \\
& \quad+\frac{2 b_{1} b_{2}(1-\alpha)}{\alpha\left(b_{2}-b_{1}\right)}(Y \circ \Lambda)(k) \\
& \quad \leq \frac{b_{1} b_{2} B(\alpha)}{\alpha\left(b_{2}-b_{1}\right)}\left[\left({ }_{1 / b_{2}}^{C F} I^{\alpha} Y \circ \Lambda\right)(k)+\left(\begin{array}{l}
C F \\
1 / b_{1}
\end{array} I^{\alpha} Y \circ \Lambda\right)(k)\right] \\
& \quad \leq \frac{2 b_{1} b_{2}(1-\alpha)}{\alpha\left(b_{2}-b_{1}\right)}(Y \circ \Lambda)(k)+\frac{\left[Y\left(b_{1}\right)+Y\left(b_{2}\right)\right]}{2}-\frac{1}{6} \psi\left(\left|\frac{1}{b_{1}}-\frac{1}{b_{2}}\right|\right) .
\end{aligned}
$$

Corollary 17. Under the assumptions of Theorem 15, if $Y$ is a strongly harmonic convex function, then

$$
\begin{aligned}
& Y\left(\frac{2 b_{1} b_{2}}{b_{1}+b_{2}}\right)+\frac{\left(b_{2}-b_{1}\right)^{2}}{24\left(b_{1} b_{2}\right)^{2}}+\frac{2 b_{1} b_{2}(1-\alpha)}{\alpha\left(b_{2}-b_{1}\right)}(Y \circ \Lambda)(k) \\
& \quad \leq \frac{b_{1} b_{2} B(\alpha)}{\alpha\left(b_{2}-b_{1}\right)}\left[\left(\begin{array}{l}
C F \\
1 / b_{2}
\end{array} I^{\alpha} Y \circ \Lambda\right)(k)+\left(\begin{array}{l}
C F \\
1 / b_{1}
\end{array} I^{\alpha} Y \circ \Lambda\right)(k)\right] \\
& \quad \leq \frac{2 b_{1} b_{2}(1-\alpha)}{\alpha\left(b_{2}-b_{1}\right)}(Y \circ \Lambda)(k)+\frac{\left[Y\left(b_{1}\right)+Y\left(b_{2}\right)\right]}{2}-\frac{\delta}{12}\left(\frac{b_{2}-b_{1}}{b_{1} b_{2}}\right)^{2} .
\end{aligned}
$$


Corollary 18. Under the assumptions of Theorem 15, if $Y$ is a uniform harmonic s-convex function, then

$$
\begin{aligned}
& 2^{s-1} Y\left(\frac{2 b_{1} b_{2}}{b_{1}+b_{2}}\right)+\frac{b_{1} b_{2}}{8\left(b_{2}-b_{1}\right)} \int_{\left(b_{1}-b_{2}\right) / b_{1} b_{2}}^{\left(b_{2}-b_{1}\right) / b_{1} b_{2}} \psi(|i|) d i \\
& +\frac{2 b_{1} b_{2}(1-\alpha)}{\alpha\left(b_{2}-b_{1}\right)}(Y \circ \Lambda)(k) \\
& \leq \frac{b_{1} b_{2} B(\alpha)}{\alpha\left(b_{2}-b_{1}\right)}\left[\left(\begin{array}{l}
C F \\
1 / b_{2}
\end{array} I^{\alpha} Y \circ \Lambda\right)(k)+\left({ }_{1 / b_{1}}^{C F} I^{\alpha} Y \circ \Lambda\right)(k)\right] \\
& \leq \frac{2 b_{1} b_{2}(1-\alpha)}{\alpha\left(b_{2}-b_{1}\right)}(Y \circ \Lambda)(k)+\frac{\left[Y\left(b_{1}\right)+Y\left(b_{2}\right)\right]}{1+s} \\
& -\frac{1}{(1+s)(2+s)} \psi\left(\left|\frac{1}{b_{1}}-\frac{1}{b_{2}}\right|\right) \text {. }
\end{aligned}
$$

Corollary 19. Under the assumptions of Theorem 15, if $Y$ is a strongly harmonic s-convex function, then

$$
\begin{array}{rl}
2^{s-1} & Y\left(\frac{2 b_{1} b_{2}}{b_{1}+b_{2}}\right)+\frac{\left(b_{2}-b_{1}\right)^{2}}{24\left(b_{1} b_{2}\right)^{2}}+\frac{2 b_{1} b_{2}(1-\alpha)}{\alpha\left(b_{2}-b_{1}\right)}(Y \circ \Lambda)(k) \\
\leq & \frac{b_{1} b_{2} B(\alpha)}{\alpha\left(b_{2}-b_{1}\right)}\left[\left(\begin{array}{l}
C F \\
1 / b_{2}
\end{array} I^{\alpha} Y \circ \Lambda\right)(k)+\left(\begin{array}{l}
C F \\
1 / b_{1}
\end{array} I^{\alpha} Y \circ \Lambda\right)(k)\right] \\
\leq & \frac{2 b_{1} b_{2}(1-\alpha)}{\alpha\left(b_{2}-b_{1}\right)}(Y \circ \Lambda)(k)+\frac{\left[Y\left(b_{1}\right)+Y\left(b_{2}\right)\right]}{1+s} \\
& -\frac{\delta}{2(1+s)(2+s)}\left(\frac{b_{2}-b_{1}}{b_{1} b_{2}}\right)^{2} .
\end{array}
$$

Corollary 20. Under the assumptions of Theorem 15, if $Y$ is a GLD type of harmonic s-convex functions, then

$$
\begin{aligned}
& 2^{-s-1} Y\left(\frac{2 b_{1} b_{2}}{b_{1}+b_{2}}\right)+\frac{b_{1} b_{2}}{8\left(b_{2}-b_{1}\right)} \int_{\left(b_{1}-b_{2}\right) / b_{1} b_{2}}^{\left(b_{2}-b_{1}\right) / b_{1} b_{2}} \psi(|i|) d i \\
& +\frac{2 b_{1} b_{2}(1-\alpha)}{\alpha\left(b_{2}-b_{1}\right)}(Y \circ \Lambda)(k) \\
& \leq \frac{b_{1} b_{2} B(\alpha)}{\alpha\left(b_{2}-b_{1}\right)}\left[\left({ }_{1 / b_{2}}^{C F} I^{\alpha} Y \circ \Lambda\right)(k)+\left({ }_{1 / b_{1}}^{C F} I^{\alpha} Y \circ \Lambda\right)(k)\right] \\
& \leq \frac{2 b_{1} b_{2}(1-\alpha)}{\alpha\left(b_{2}-b_{1}\right)}(Y \circ \Lambda)(k)+\frac{\left[Y\left(b_{1}\right)+Y\left(b_{2}\right)\right]}{1-s} \\
& -\frac{1}{(1-s)(2-s)} \psi\left(\left|\frac{1}{b_{1}}-\frac{1}{b_{2}}\right|\right) \text {. }
\end{aligned}
$$

2.4. Dragomir-Agarwal Type of Inequalities. In this section, we derive the Dragomir-Agarwal type of inequalities using the concept of uniform harmonic $\chi$-convex functions. For this, we first derive a new lemma which will be used as an auxiliary result in obtaining our next results.
Lemma 21. Let $Y:\left[b_{1}, b_{2}\right] \subset \mathbb{R} \longrightarrow \mathbb{R}$ be a differentiable function on $\left(b_{1}, b_{2}\right)$ with $b_{1}<b_{2}$. If $Y^{\prime} \in t_{1}\left[b_{1}, b_{2}\right], \alpha \in[0,1]$, and also $k \in\left[b_{1}, b_{2}\right]$, then we have

$$
\begin{aligned}
& \frac{Y\left(b_{1}\right)+Y\left(b_{2}\right)}{2}+\frac{2 b_{1} b_{2}}{\alpha\left(b_{2}-b_{1}\right)}(Y \circ \Lambda)(k)-\frac{b_{1} b_{2} B(\alpha)}{\alpha\left(b_{2}-b_{1}\right)} \\
& \times\left[\left(\begin{array}{l}
C F \\
1 / b_{2}
\end{array} I^{\alpha} Y \circ \Lambda\right)(k)+\left({ }_{1 / b_{1}}^{C F} I^{\alpha} Y \circ \Lambda\right)(k)\right] \\
& =\frac{b_{1} b_{2}\left(b_{2}-b_{1}\right)}{2} \int_{0}^{1} \frac{1-2 i}{\left((1-i) b_{1}+i b_{2}\right)^{2}} Y^{\prime}\left(\frac{b_{1} b_{2}}{(1-i) b_{1}+i b_{2}}\right) d i \text {, }
\end{aligned}
$$

where $\Lambda(x)=1 / x$.

Proof. Let us consider

$$
\begin{aligned}
& \int_{0}^{1} \frac{1-2 i}{\left((1-i) b_{1}+i b_{2}\right)^{2}} Y^{\prime}\left(\frac{b_{1} b_{2}}{(1-i) b_{1}+i b_{2}}\right) d i \\
& \quad=\frac{Y\left(b_{1}\right)+Y\left(b_{2}\right)}{b_{1} b_{2}\left(b_{2}-b_{1}\right)}-\frac{2}{b_{1} b_{2}\left(b_{2}-b_{1}\right)} \int_{0}^{1} Y\left(\frac{b_{1} b_{2}}{(1-i) b_{1}+i b_{2}}\right) d i \\
& \quad=\frac{Y\left(b_{1}\right)+Y\left(b_{2}\right)}{b_{1} b_{2}\left(b_{2}-b_{1}\right)}-\frac{2}{\left(b_{2}-b_{1}\right)^{2}} \int_{1 / b_{2}}^{1 / b_{1}} Y \circ \Lambda(x) d x
\end{aligned}
$$

Multiplying both sides by $\alpha\left(b_{2}-b_{1}\right)^{2} / 2 B(\alpha)$ and subtracting $(2(1-\alpha) / B(\alpha))(Y \circ \Lambda)(k)$, we have

$$
\begin{aligned}
& \frac{\alpha\left(b_{2}-b_{1}\right)^{2}}{2 B(\alpha)} \int_{0}^{1} \frac{1-2 i}{\left((1-i) b_{1}+i b_{2}\right)^{2}} Y^{\prime}\left(\frac{b_{1} b_{2}}{(1-i) b_{1}+i b_{2}}\right) d i \\
& -\frac{2(1-\alpha)}{B(\alpha)}(Y \circ \Lambda)(k) \\
& =\frac{\alpha\left(b_{2}-b_{1}\right)\left[Y\left(b_{1}\right)+Y\left(b_{2}\right)\right]}{2 b_{1} b_{2} B(\alpha)}-\frac{2(1-\alpha)}{B(\alpha)}(Y \circ \Lambda)(k) \\
& -\frac{\alpha}{B(\alpha)} \int_{1 / b_{2}}^{1 / b_{1}} Y \circ \Lambda(x) d x=\frac{\alpha\left(b_{2}-b_{1}\right)\left[Y\left(b_{1}\right)+Y\left(b_{2}\right)\right]}{2 b_{1} b_{2} B(\alpha)} \\
& -\frac{2(1-\alpha)}{B(\alpha)}(Y \circ \Lambda)(k)-\frac{\alpha}{B(\alpha)}\left[\int_{1 / b_{2}}^{k} Y \circ \Lambda(x) d x+\int_{k}^{1 / b_{1}} Y \circ \Lambda(x) d x\right] \\
& =\frac{\alpha\left(b_{2}-b_{1}\right)\left[Y\left(b_{1}\right)+Y\left(b_{2}\right)\right]}{2 b_{1} b_{2} B(\alpha)}-\left[\frac{(1-\alpha)}{B(\alpha)}(Y \circ \Lambda)(k)+\frac{\alpha}{B(\alpha)} \int_{1 / b_{2}}^{k} Y\right. \\
& \left.\circ \Lambda(x) d x+\frac{(1-\alpha)}{B(\alpha)}(Y \circ \Lambda)(k)+\frac{\alpha}{B(\alpha)} \int_{k}^{1 / b_{1}} Y \circ \Lambda(x) d x\right] \\
& =\frac{\alpha\left(b_{2}-b_{1}\right)\left[Y\left(b_{1}\right)+Y\left(b_{2}\right)\right]}{2 b_{1} b_{2} B(\alpha)}-\left[\left({ }_{1 / b_{2}}^{C F} I^{\alpha} Y \circ \Lambda\right)(k)+\left({ }_{1 / b_{1}}^{C F} I^{\alpha} Y \circ \Lambda\right)(k)\right] .
\end{aligned}
$$

This completes the proof. 
Theorem 22. If $Y:\left[b_{1}, b_{2}\right] \longrightarrow \mathbb{R}$ is a uniform harmonic $\chi$ -convex function with $b_{1}<b_{2}$ and $i \in[0,1]$, then we have

$$
\begin{aligned}
& \mid \frac{Y\left(b_{1}\right)+Y\left(b_{2}\right)}{2}+\frac{2 b_{1} b_{2}}{\alpha\left(b_{2}-b_{1}\right)}(Y \circ \Lambda)(k)-\frac{b_{1} b_{2} B(\alpha)}{\alpha\left(b_{2}-b_{1}\right)} \\
& \times\left[\left({ }_{1 / b_{2}}^{C F} I^{\alpha} Y \circ \Lambda\right)(k)+\left({ }_{1 / b_{1}}^{C F} I^{\alpha} Y \circ \Lambda\right)(k)\right] \mid \\
& \leq \frac{b_{1} b_{2}\left(b_{2}-b_{1}\right)}{2} \int_{0}^{1}\left|\frac{1-2 i}{\left((1-i) b_{1}+i b_{2}\right)^{2}}\right|\left[\chi(i)\left|Y^{\prime}\left(b_{1}\right)\right|\right. \\
& \left.+\chi(1-i)\left|Y^{\prime}\left(b_{2}\right)\right|-(1-i) \chi(i) \psi\left(\left|\frac{1}{b_{2}}-\frac{1}{b_{1}}\right|\right)\right] d i .
\end{aligned}
$$

Proof. Using Lemma 21, modulus property, and uniform harmonic $\chi$-convexity of $\left|Y^{\prime}\right|$, we have

$$
\begin{aligned}
& \mid \frac{Y\left(b_{1}\right)+Y\left(b_{2}\right)}{2}+\frac{2 b_{1} b_{2}}{\alpha\left(b_{2}-b_{1}\right)}(Y \circ \Lambda)(k)-\frac{b_{1} b_{2} B(\alpha)}{\alpha\left(b_{2}-b_{1}\right)} \\
& \times\left[\left(\begin{array}{l}
C F \\
1 / b_{2}
\end{array} I^{\alpha} Y \circ \Lambda\right)(k)+\left({ }_{1 / b_{1}}^{C F} I^{\alpha} Y \circ \Lambda\right)(k)\right] \mid \\
& \leq \frac{b_{1} b_{2}\left(b_{2}-b_{1}\right)}{2} \int_{0}^{1}\left|\frac{1-2 i}{\left((1-) b_{1}+i b_{2}\right)^{2}}\right|\left|Y^{\prime}\left(\frac{b_{1} b_{2}}{(1-i) b_{1}+i b_{2}}\right)\right| d i \\
& \leq \frac{b_{1} b_{2}\left(b_{2}-b_{1}\right)}{2} \int_{0}^{1}\left|\frac{1-2 i}{\left((1-i) b_{1}+i b_{2}\right)^{2}}\right|\left[\chi(i)\left|Y^{\prime}\left(b_{1}\right)\right|\right. \\
& \left.+\chi(1-i)\left|Y^{\prime}\left(b_{2}\right)\right|-(1-i) \chi(i) \psi\left(\left|\frac{1}{b_{2}}-\frac{1}{b_{1}}\right|\right)\right] d i .
\end{aligned}
$$

This completes the proof.

Now, we discuss some special cases of Theorem 22:

(i) If we take $\chi(i)=1$ in Theorem 22, then we have the result for uniform harmonic $P$-convex functions

(ii) If we take $\chi(i)=i$ in Theorem 22 , then we have the result for uniform harmonic convex functions

(iii) If we take $\chi(i)=i^{s}$ in Theorem 22 and by similar proceedings as in the above corollaries, then we have the result for uniform harmonic $s$-convex functions

Corollary 23. Under the assumptions of Theorem 22, if $\left|Y^{\prime}\right|$ is a uniform harmonic P-convex function, then

$$
\begin{aligned}
& \mid \frac{Y\left(b_{1}\right)+Y\left(b_{2}\right)}{2}+\frac{2 b_{1} b_{2}}{\alpha\left(b_{2}-b_{1}\right)}(Y \circ \Lambda)(k)-\frac{b_{1} b_{2} B(\alpha)}{\alpha\left(b_{2}-b_{1}\right)} \\
& \quad \times\left[\left({ }_{1 / b_{2}}^{C F} I^{\alpha} Y \circ \Lambda\right)(k)+\left({ }_{1 / b_{1}} I^{\alpha} Y \circ \Lambda\right)(k)\right] \mid \\
& \leq \\
& \quad \times\left[\left|Y_{1}^{\prime} b_{2}\left(b_{2}-b_{1}\right)\right|+\left|Y^{\prime}\left(b_{2}\right)\right|-(1-i) \psi\left(\left|\frac{1}{b_{2}}-\frac{1}{b_{1}}\right|\right)\right] d i \\
& \quad+\int_{0}^{1} \frac{2 i-1}{\left((1-i) b_{1}+i b_{2}\right)^{2}} \\
& \leq \frac{b_{1} b_{2}\left(b_{2}-b_{1}\right)}{2}\left[s_{1}\left[\left|Y^{\prime}\left(b_{1}\right)\right|+\left|Y^{\prime}\left(b_{2}\right)\right|\right]-s_{2} \psi\left(\left|\frac{1}{b_{2}}-\frac{1}{b_{1}}\right|\right)\right],
\end{aligned}
$$

where

$$
\begin{aligned}
s_{1}= & \frac{b_{1}^{-2}}{2} \mathscr{F}_{1}\left(2,1,3, \frac{1}{2}\left(1-\frac{b_{2}}{b_{1}}\right)\right)+b_{1}^{-2} \mathscr{F}_{1}\left(2,2,3,1-\frac{b_{2}}{b_{1}}\right)-\frac{1}{b_{1} b_{2}}, \\
s_{2}= & \frac{b_{1}^{-2}}{2} \mathscr{F}_{1}\left(2,1,3, \frac{1}{2}\left(1-\frac{b_{2}}{b_{1}}\right)\right)-\frac{b_{1}^{-2}}{2} \mathscr{F}_{1}\left(2,2,4, \frac{1}{2}\left(1-\frac{b_{2}}{b_{1}}\right)\right) \\
& +\frac{b_{1}^{-2}}{3} \mathscr{F}_{1}\left(2,2,4,1-\frac{b_{2}}{b_{1}}\right)-\frac{b_{1}^{-2}}{2} \mathscr{F}_{1}\left(2,1,3,1-\frac{b_{2}}{b_{1}}\right) .
\end{aligned}
$$

Corollary 24. Under the assumptions of Theorem 22, if $\left|Y^{\prime}\right|$ is a uniform harmonic convex function, then

$$
\begin{aligned}
& \mid \frac{Y\left(b_{1}\right)+Y\left(b_{2}\right)}{2}+\frac{2 b_{1} b_{2}}{\alpha\left(b_{2}-b_{1}\right)}(Y \circ \Lambda)(k)-\frac{b_{1} b_{2} B(\alpha)}{\alpha\left(b_{2}-b_{1}\right)} \\
& \times\left[\left(\begin{array}{l}
C F \\
1 / b_{2}
\end{array} I^{\alpha} Y \circ \Lambda\right)(k)+\left({ }_{1 / b_{1}}^{C F} I^{\alpha} Y \circ \Lambda\right)(k)\right] \mid \\
& \leq \frac{b_{1} b_{2}\left(b_{2}-b_{1}\right)}{2}\left[2 \int_{0}^{1 / 2} \frac{1-2 i}{\left((1-i) b_{1}+i b_{2}\right)^{2}}\right. \\
& \times\left[i\left|Y^{\prime}\left(b_{1}\right)\right|+(1-i)\left|Y^{\prime}\left(b_{2}\right)\right|-(1-i) i \psi\left(\left|\frac{1}{b_{2}}-\frac{1}{b_{1}}\right|\right)\right] d i \\
& +\int_{0}^{1} \frac{2 i-1}{\left((1-i) b_{1}+i b_{2}\right)^{2}}\left[i\left|Y^{\prime}\left(b_{1}\right)\right|+(1-i)\left|Y^{\prime}\left(b_{2}\right)\right|\right. \\
& \left.\left.-(1-i) i \psi\left(\left|\frac{1}{b_{2}}-\frac{1}{b_{1}}\right|\right)\right] d i\right] \\
& \leq \frac{b_{1} b_{2}\left(b_{2}-b_{1}\right)}{2}\left[s_{3}\left|Y^{\prime}\left(b_{1}\right)\right|+s_{4}\left|Y^{\prime}\left(b_{2}\right)\right|-s_{5} \psi\left(\left|\frac{1}{b_{2}}-\frac{1}{b_{1}}\right|\right)\right] \text {, }
\end{aligned}
$$

where

$$
\begin{aligned}
s_{3}= & \frac{b_{1}^{-2}}{6} \mathscr{F}_{1}\left(2,2,4, \frac{1}{2}\left(1-\frac{b_{2}}{b_{1}}\right)\right)+\frac{2 b_{1}^{-2}}{3} \mathscr{F}_{1} \\
& \cdot\left(2,3,4,1-\frac{b_{2}}{b_{1}}\right)-\frac{b_{1}^{-2}}{2} \mathscr{F}_{1}\left(2,2,3,1-\frac{b_{2}}{b_{1}}\right), \\
s_{4}= & \frac{b_{1}^{-2}}{2} \mathscr{F}_{1}\left(2,1,3, \frac{1}{2}\left(1-\frac{b_{2}}{b_{1}}\right)\right)-\frac{b_{1}^{-2}}{6} \mathscr{F}_{1} \\
& \cdot\left(2,2,4, \frac{1}{2}\left(1-\frac{b_{2}}{b_{1}}\right)\right)+\frac{b_{1}{ }^{-2}}{3} \mathscr{F}_{1}\left(2,2,4,1-\frac{b_{2}}{b_{1}}\right) \\
& -\frac{b_{1}{ }^{-2}}{2} \mathscr{F}_{1}\left(2,1,3,1-\frac{b_{2}}{b_{1}}\right), \\
& \frac{b_{1}{ }^{-2} \mathscr{F}_{1}\left(2,2,4, \frac{1}{2}\left(1-\frac{b_{2}}{b_{1}}\right)\right)-\frac{b_{1}{ }^{-2}}{48} \mathscr{F}_{1}}{12}{ }_{2} \\
& \cdot\left(2,3,5, \frac{1}{2}\left(1-\frac{b_{2}}{b_{1}}\right)\right)+\frac{b_{1}{ }^{-2}}{6} \mathscr{F}_{1}\left(2,3,5,1-\frac{b_{2}}{b_{1}}\right) \\
& -\frac{b_{1}{ }^{-2}}{6} \mathscr{F}_{1}\left(2,2,4,1-\frac{b_{2}}{b_{1}}\right) .
\end{aligned}
$$


Remark 25. In Corollary 24, if we take

(1) $\psi=\delta i^{2}$, then we can obtain the result for a strongly harmonic convex function

(2) $\psi=-\delta|i|$, then we can obtain the result for an approximate harmonic convex function

(3) $\psi=-\delta|i|^{p}$, then we can obtain the result for an approximate harmonic convex function of order $p>0$

Corollary 26. Under the assumptions of Theorem 22, if $\left|Y^{\prime}\right|$ is a uniform harmonic s-convex function, then

$$
\begin{aligned}
& \mid \frac{Y\left(b_{1}\right)+Y\left(b_{2}\right)}{2}+\frac{2 b_{1} b_{2}}{\alpha\left(b_{2}-b_{1}\right)}(Y \circ \Lambda)(k)-\frac{b_{1} b_{2} B(\alpha)}{\alpha\left(b_{2}-b_{1}\right)} \\
& \times\left[\left(\begin{array}{l}
C F \\
1 / b_{2}
\end{array} I^{\alpha} Y \circ \Lambda\right)(k)+\left(\begin{array}{l}
C F \\
1 / b_{1}
\end{array} I^{\alpha} Y \circ \Lambda\right)(k)\right] \mid \\
& \leq \frac{b_{1} b_{2}\left(b_{2}-b_{1}\right)}{2}\left[s_{6}\left|Y^{\prime}\left(b_{1}\right)\right|+s_{7}\left|Y^{\prime}\left(b_{2}\right)\right|-s_{8} \psi\left(\left|\frac{1}{b_{2}}-\frac{1}{b_{1}}\right|\right)\right],
\end{aligned}
$$

where

$$
\begin{aligned}
& s_{6}=\frac{b_{1}{ }^{-2}}{(s+1)(s+2)_{2}} \mathscr{F}_{1}\left(2, s+1, s+3, \frac{1}{2}\left(1-\frac{b_{2}}{b_{1}}\right)\right) \\
& +\frac{2 b_{1}{ }^{-2}}{s+2} \mathscr{F}_{1}\left(2, s+2, s+3,1-\frac{b_{2}}{b_{1}}\right)-\frac{b_{1}{ }^{-2}}{s+12} \mathscr{F}_{1} \\
& \cdot\left(2, s+1, s+2,1-\frac{b_{2}}{b_{1}}\right) \text {, } \\
& s_{7}=\frac{b_{1}^{-2}}{2} \mathscr{F}_{1}\left(2,1,3, \frac{1}{2}\left(1-\frac{b_{2}}{b_{1}}\right)\right)-\frac{b_{1}^{-2}}{2^{s}(s+1)(s+2)_{2}} \mathscr{F}_{1} \\
& \cdot\left(2, s+1, s+3, \frac{1}{2}\left(1-\frac{b_{2}}{b_{1}}\right)\right)+\frac{2 b_{1}^{-2}}{(s+1)(s+2)_{2}} \mathscr{F}_{1} \\
& \cdot\left(2,2, s+3,1-\frac{b_{2}}{b_{1}}\right)-\frac{b_{1}{ }^{-2}}{s+1} \mathscr{F}_{1}\left(2,1, s+2,1-\frac{b_{2}}{b_{1}}\right) \text {, } \\
& s_{8}=\frac{b_{1}{ }^{-2}}{2^{s}(1+s)(2+s)_{2}} \mathscr{F}_{1}\left(2, s+1, s+3, \frac{1}{2}\left(1-\frac{b_{2}}{b_{1}}\right)\right) \\
& -\frac{b_{1}{ }^{-2}}{2^{1+s}(s+2)(s+3)_{2}} \mathscr{F}_{1}\left(2, s+2, s+4, \frac{1}{2}\left(1-\frac{b_{2}}{b_{1}}\right)\right) \\
& +\frac{2 b_{1}^{-2}}{(s+3)(s+2)_{2}} \mathscr{F}_{1}\left(2, s+2, s+4,1-\frac{b_{2}}{b_{1}}\right) \\
& -\frac{b_{1}{ }^{-2}}{(s+1)(s+2)_{2}} \mathscr{F}_{1}\left(2, s+2, s+3,1-\frac{b_{2}}{b_{1}}\right) \text {. }
\end{aligned}
$$

\section{Remark 27. In Corollary 26, if we take}

(1) $\psi=\delta i^{2}$, then we can obtain the result for a strongly harmonic $s$-convex function
(2) $\psi=-\delta|i|$, then we can obtain the result for an approximate harmonic $s$-convex function

(3) $\psi=-\delta|i|^{p}$, then we can obtain the result for an approximate harmonic $s$-convex function of order $p>0$

Theorem 28. If $Y:\left[b_{1}, b_{2}\right] \longrightarrow \mathbb{R}$ is a uniform harmonic $\chi$-convex function with $b_{1}<b_{2}$ and $i \in[0,1]$, then we have

$$
\begin{aligned}
& \mid \frac{Y\left(b_{1}\right)+Y\left(b_{2}\right)}{2}+\frac{2 b_{1} b_{2}}{\alpha\left(b_{2}-b_{1}\right)}(Y \circ \Lambda)(k)-\frac{b_{1} b_{2} B(\alpha)}{\alpha\left(b_{2}-b_{1}\right)} \\
& \quad \times\left[\left({ }_{1 / b_{2}}^{C F} I^{\alpha} Y \circ \Lambda\right)(k)+\left({ }_{1 / b_{1}}^{C F} I^{\alpha} Y \circ \Lambda\right)(k)\right] \mid \\
& \leq \frac{b_{1} b_{2}\left(b_{2}-b_{1}\right)}{2}\left(\frac{1}{p+1}\right)^{1 / p}\left[\int_{0}^{1} \frac{1}{\left((1-i) b_{1}+i b_{2}\right)^{2}}\right. \\
& \quad \times\left(\chi(i)\left|Y^{\prime}\left(b_{1}\right)\right|^{q}+\chi(1-i)\left|Y^{\prime}\left(b_{2}\right)\right|^{q}\right. \\
& \left.\left.\quad-(1-i) \chi(i) \psi\left(\left|\frac{1}{b_{2}}-\frac{1}{b_{1}}\right|\right)\right)\right]^{1 / q} d i .
\end{aligned}
$$

Proof. Using Lemma 21, modulus property, and uniform harmonic $\chi$-convexity of $\left|Y^{\prime}\right|^{q}$, then we have

$$
\begin{aligned}
& \mid \frac{Y\left(b_{1}\right)+Y\left(b_{2}\right)}{2}+\frac{2 b_{1} b_{2}}{\alpha\left(b_{2}-b_{1}\right)}(Y \circ \Lambda)(k)-\frac{b_{1} b_{2} B(\alpha)}{\alpha\left(b_{2}-b_{1}\right)} \\
& \times\left[\left(\begin{array}{l}
C F \\
1 / b_{2}
\end{array} I^{\alpha} Y \circ \Lambda\right)(k)+\left(\begin{array}{l}
C F \\
1 / b_{1}
\end{array} I^{\alpha} Y \circ \Lambda\right)(k)\right] \\
& \leq \frac{b_{1} b_{2}\left(b_{2}-b_{1}\right)}{2} \int_{0}^{1}\left|\frac{1-2 i}{\left((1-i) b_{1}+i b_{2}\right)^{2}}\right|\left|Y^{\prime}\left(\frac{b_{1} b_{2}}{(1-i) b_{1}+i b_{2}}\right)\right| d i \\
& \leq \frac{b_{1} b_{2}\left(b_{2}-b_{1}\right)}{2}\left(\int_{0}^{1}|1-2 i|^{p}\right)^{1 / p}\left[\int_{0}^{1} \frac{1}{\left((1-i) b_{1}+i b_{2}\right)^{2 q}}\right. \\
& \times\left(\chi(i)\left|Y^{\prime}\left(b_{1}\right)\right|^{q}+\chi(1-i)\left|Y^{\prime}\left(b_{2}\right)\right|^{q}\right. \\
& \left.\left.-(1-i) \chi(i) \psi\left(\left|\frac{1}{b_{2}}-\frac{1}{b_{1}}\right|\right)\right)\right]^{1 / q} d i
\end{aligned}
$$

This completes the proof.

Now, we discuss some special cases of Theorem 28:

(i) If we take $\chi(i)=1$ in Theorem 28, then we have the result for uniform harmonic $P$-convex functions

(ii) If we take $\chi(i)=i$ in Theorem 22, then we have the result for uniform harmonic convex functions

(iii) If we take $\chi(i)=i^{s}$ in Theorem 22, then we have the result for uniform harmonic s-convex functions 
Corollary 29. Under the assumptions of Theorem 28, if $\left|Y^{\prime}\right|$ is a uniform harmonic P-convex function, then

$$
\begin{aligned}
& \mid \frac{Y\left(b_{1}\right)+Y\left(b_{2}\right)}{2}+\frac{2 b_{1} b_{2}}{\alpha\left(b_{2}-b_{1}\right)}(Y \circ \Lambda)(k)-\frac{b_{1} b_{2} B(\alpha)}{\alpha\left(b_{2}-b_{1}\right)} \\
& \times\left[\left({ }_{1 / b_{2}}^{C F} I^{\alpha} Y \circ \Lambda\right)(k)+\left({ }_{1 / b_{1}}^{C F} I^{\alpha} Y \circ \Lambda\right)(k)\right] \\
& \leq \frac{b_{1} b_{2}\left(b_{2}-b_{1}\right)}{2}\left(\frac{1}{p+1}\right)^{1 / p} \\
& \times\left[\frac{\left(b_{2}^{1-2 q}-b_{1}^{1-2 q}\right)\left(\left|Y^{\prime}\left(b_{1}\right)\right|^{q}+\left|Y^{\prime}\left(b_{2}\right)\right|^{q}\right)}{\left(b_{2}-b_{1}\right)(1-2 q)}\right. \\
& \left.-\frac{b_{1}}{2} \mathscr{F}_{1}\left(2 q, 1,3,\left(1-\frac{b_{2}}{b_{1}}\right)\right) \psi\left(\left|\frac{1}{b_{2}}-\frac{1}{b_{1}}\right|\right)\right]^{1 / q} \text {. }
\end{aligned}
$$

Corollary 30. Under the assumptions of Theorem 22, if $\left|Y^{\prime}\right|$ is a uniform harmonic convex function, then

$$
\begin{aligned}
& \mid \frac{Y\left(b_{1}\right)+Y\left(b_{2}\right)}{2}+\frac{2 b_{1} b_{2}}{\alpha\left(b_{2}-b_{1}\right)}(Y \circ \Lambda)(k)-\frac{b_{1} b_{2} B(\alpha)}{\alpha\left(b_{2}-b_{1}\right)} \\
& \quad \times\left[\left(\begin{array}{c}
C F \\
1 / b_{2}
\end{array} I^{\alpha} Y \circ \Lambda\right)(k)+\left({ }_{1 / b_{1}}^{C F} I^{\alpha} Y \circ \Lambda\right)(k)\right] \mid \\
& \leq \frac{b_{1} b_{2}\left(b_{2}-b_{1}\right)}{2}\left(\frac{1}{p+1}\right)^{1 / p}\left[\int_{0}^{1} \frac{1}{\left((1-i) b_{1}+i b_{2}\right)^{2 q}}\right. \\
& \left.\quad \times\left(i\left|Y^{\prime}\left(b_{1}\right)\right|+(1-i)\left|Y^{\prime}\left(b_{2}\right)\right|-(1-i) i \psi\left(\left|\frac{1}{b_{2}}-\frac{1}{b_{1}}\right|\right)\right) d i\right] \\
& \leq \frac{b_{1} b_{2}\left(b_{2}-b_{1}\right)}{2}\left(\frac{1}{p+1}\right)^{1 / p} \\
& \quad \times\left[s_{9}\left|Y^{\prime}\left(b_{1}\right)\right|^{q}+s_{10}\left|Y^{\prime}\left(b_{2}\right)\right|^{q}-s_{11} \psi\left(\left|\frac{1}{b_{2}}-\frac{1}{b_{1}}\right|\right)\right]^{1 / q},
\end{aligned}
$$

where

$$
\begin{gathered}
s_{9}=\frac{b_{1}^{-2 q}}{2} \mathscr{F}_{1}\left(2 q, 2,3,\left(1-\frac{b_{2}}{b_{1}}\right)\right), \\
s_{10}=\frac{b_{1}^{-2 q}}{2} \mathscr{F}_{1}\left(2 q, 1,3,\left(1-\frac{b_{2}}{b_{1}}\right)\right), \\
s_{11}=\frac{b_{1}^{-2 q}}{2} \mathscr{F}_{1}\left(2 q, 2,4,\left(1-\frac{b_{2}}{b_{1}}\right)\right) .
\end{gathered}
$$

Remark 31. In Corollary 30, if we take

(1) $\psi=\delta i^{2}$, then we can obtain the result for a strongly harmonic convex function

(2) $\psi=-\delta|i|$, then we can obtain the result for an approximate harmonic convex function
(3) $\psi=-\delta|i|^{p}$, then we can obtain the result for an approximate harmonic convex function of order $p>0$

Corollary 32. Under the assumptions of Theorem 22, if $\left|Y^{\prime}\right|$ is a uniform harmonic s-convex function, then

$$
\begin{aligned}
& \mid \frac{Y\left(b_{1}\right)+Y\left(b_{2}\right)}{2}+\frac{2 b_{1} b_{2}}{\alpha\left(b_{2}-b_{1}\right)}(Y \circ \Lambda)(k)-\frac{b_{1} b_{2} B(\alpha)}{\alpha\left(b_{2}-b_{1}\right)} \\
& \quad \times\left[\left(C_{1 / b_{2}}^{C F} I^{\alpha} Y \circ \Lambda\right)(k)+\left(\begin{array}{l}
C F \\
1 / b_{1}
\end{array} I^{\alpha} Y \circ \Lambda\right)(k)\right] \mid \\
& \leq \frac{b_{1} b_{2}\left(b_{2}-b_{1}\right)}{2}\left(\frac{1}{p+1}\right)^{1 / p}\left[\int_{0}^{1} \frac{1}{\left((1-i) b_{1}+i b_{2}\right)^{2 q}}\right. \\
& \left.\quad \times\left(i^{s}\left|Y^{\prime}\left(b_{1}\right)\right|+(1-i)^{s}\left|Y^{\prime}\left(b_{2}\right)\right|-(1-i) i^{s} \psi\left(\left|\frac{1}{b_{2}}-\frac{1}{b_{1}}\right|\right)\right) d i\right] \\
& \leq \frac{b_{1} b_{2}\left(b_{2}-b_{1}\right)}{2}\left(\frac{1}{p+1}\right)^{1 / p}\left[s_{12}\left|Y^{\prime}\left(b_{1}\right)\right|^{q}+s_{13}\left|Y^{\prime}\left(b_{2}\right)\right|^{q}-s_{14} \psi\right. \\
& \left.\quad \times\left(\left|\frac{1}{b_{2}}-\frac{1}{b_{1}}\right|\right)\right]^{1 / q},
\end{aligned}
$$

where

$$
\begin{aligned}
s_{12} & =\frac{b_{1}^{-2 q}}{s+1} \mathscr{F}_{1}\left(2 q, s+1, s+2,\left(1-\frac{b_{2}}{b_{1}}\right)\right), \\
s_{13} & =\frac{b_{1}^{-2 q}}{s+1} \mathscr{F}_{1}\left(2 q, 1, s+2,\left(1-\frac{b_{2}}{b_{1}}\right)\right), \\
s_{14} & =\frac{b_{1}^{-2 q}}{(s+2)(s+1)_{2}} \mathscr{F}_{1}\left(2 q, s+1, s+3,\left(1-\frac{b_{2}}{b_{1}}\right)\right) .
\end{aligned}
$$

Remark 33. In Corollary 32, if we take

(1) $\psi=\delta i^{2}$, then we can obtain the result for a strongly harmonic $s$-convex function

(2) $\psi=-\delta|i|$, then we can obtain the result for an approximate harmonic $s$-convex function

(3) $\psi=-\delta|i|^{p}$, then we can obtain the result for an approximate harmonic $s$-convex function of order $p>0$

Theorem 34. If $Y:\left[b_{1}, b_{2}\right] \longrightarrow \mathbb{R}$ is a uniform harmonic $\chi$-convex function with $b_{1}<b_{2}, 1 / p+1 / q=1$, and $i \in[0,1]$, then

$$
\begin{aligned}
& \mid \frac{Y\left(b_{1}\right)+Y\left(b_{2}\right)}{2}+\frac{2 b_{1} b_{2}}{\alpha\left(b_{2}-b_{1}\right)}(Y \circ \Lambda)(k)-\frac{b_{1} b_{2} B(\alpha)}{\alpha\left(b_{2}-b_{1}\right)} \\
& \times\left[\left({ }_{1 / b_{2}}^{C F} I^{\alpha} Y \circ \Lambda\right)(k)+\left({ }_{1 / b_{1}}^{C F} I^{\alpha} Y \circ \Lambda\right)(k)\right] \\
& \leq \frac{b_{1} b_{2}\left(b_{2}-b_{1}\right)}{2} s_{15}^{1 / p}\left(\int _ { 0 } ^ { 1 } | \frac { 1 - 2 i } { ( ( 1 - i ) b _ { 1 } + i b _ { 2 } ) ^ { 2 } } | \left[\chi(i)\left|Y^{\prime}\left(b_{1}\right)\right|^{q}\right.\right. \\
& \left.\left.+\chi(1-i)\left|Y^{\prime}\left(b_{2}\right)\right|^{q}-(1-i) \chi(i) \psi\left(\left|\frac{1}{b_{2}}-\frac{1}{b_{1}}\right|\right)\right)^{1 / q}\right] d i,
\end{aligned}
$$


where

$s_{15}=\frac{b_{1}^{-2}}{2} \mathscr{F}_{1}\left(2,1,3, \frac{1}{2}\left(1-\frac{b_{2}}{b_{1}}\right)\right)+b_{1}^{-2}{ }_{2} \mathscr{F}_{1}\left(2,2,3,1-\frac{b_{2}}{b_{1}}\right)-\frac{1}{b_{1} b_{2}}$.

Proof. Using Lemma 21, modulus property, power mean inequality, and uniform harmonic $\chi$-convexity of $\left|Y^{\prime}\right|$ and $1 / p+1 / q=1$, then we have

$$
\begin{aligned}
& \mid \frac{Y\left(b_{1}\right)+Y\left(b_{2}\right)}{2}+\frac{2 b_{1} b_{2}}{\alpha\left(b_{2}-b_{1}\right)}(Y \circ \Lambda)(k)-\frac{b_{1} b_{2} B(\alpha)}{\alpha\left(b_{2}-b_{1}\right)} \\
& \quad \times\left[\left(\begin{array}{l}
C F \\
1 / b_{2}
\end{array} I^{\alpha} Y \circ \Lambda\right)(k)+\left(\begin{array}{l}
C F \\
1 / b_{1}
\end{array} I^{\alpha} Y \circ \Lambda\right)(k)\right] \mid \\
& \leq \frac{b_{1} b_{2}\left(b_{2}-b_{1}\right)}{2}\left(\int_{0}^{1}\left|\frac{1-2 i}{\left((1-i) b_{1}+i b_{2}\right)^{2}}\right|\right)^{1 / p} \\
& \quad \times\left(\int_{0}^{1}\left|\frac{1-2 i}{\left((1-i) b_{1}+i b_{2}\right)^{2}}\right|\left|Y^{\prime}\left(\frac{b_{1} b_{2}}{(1-i) b_{1}+i b_{2}}\right)\right|^{q} d i\right)^{1 / q} \\
& \leq \frac{b_{1} b_{2}\left(b_{2}-b_{1}\right)}{2}\left(\int_{0}^{1}\left|\frac{1-2 i}{\left((1-i) b_{1}+i b_{2}\right)^{2}}\right|\right)^{1 / p} \\
& \quad \times\left(\int _ { 0 } ^ { 1 } | \frac { 1 - 2 i } { ( ( 1 - i ) b _ { 1 } + i b _ { 2 } ) ^ { 2 } } | \left[\chi(i)\left|Y^{\prime}\left(b_{1}\right)\right|^{q}\right.\right. \\
& \left.\left.\quad+\chi(1-i)\left|Y^{\prime}\left(b_{2}\right)\right|^{q}-(1-i) \chi(i) \psi\left(\left|\frac{1}{b_{2}}-\frac{1}{b_{1}}\right|\right) d i\right)^{1 / q}\right] .
\end{aligned}
$$

This completes the proof.

Now, we discuss some special cases of Theorem 34:

(i) If we take $\chi(i)=1$ in Theorem 34 and the proceedings are similar to Corollary 23 , then we have the result for uniform harmonic $P$-convex functions

(ii) If we take $\chi(i)=i$ in Theorem 34, then we have the result for uniform harmonic convex functions

(iii) If we take $\chi(i)=i^{s}$ in Theorem 34, then we have the result for uniform harmonic $s$-convex functions

Corollary 35. Under the assumptions of Theorem 34, if $\left|Y^{\prime}\right|$ is a uniform harmonic P-convex function, then

$$
\begin{aligned}
& \mid \frac{Y\left(b_{1}\right)+Y\left(b_{2}\right)}{2}+\frac{2 b_{1} b_{2}}{\alpha\left(b_{2}-b_{1}\right)}(Y \circ \Lambda)(k)-\frac{b_{1} b_{2} B(\alpha)}{\alpha\left(b_{2}-b_{1}\right)} \\
& \times\left[\left(\begin{array}{l}
C F \\
1 / b_{2}
\end{array} I^{\alpha} Y \circ \Lambda\right)(k)+\left({ }_{1 / b_{1}}^{C F} I^{\alpha} Y \circ \Lambda\right)(k)\right] \mid \\
& \leq \frac{b_{1} b_{2}\left(b_{2}-b_{1}\right)}{2} s_{15}^{1 / p}\left[s_{1}\left[\left|Y^{\prime}\left(b_{1}\right)\right|^{q}+\left|Y^{\prime}\left(b_{2}\right)\right|^{q}\right]\right. \\
& \left.-s_{2} \psi\left(\left|\frac{1}{b_{2}}-\frac{1}{b_{1}}\right|\right)\right]^{1 / q} d i
\end{aligned}
$$

where $s_{1}$ and $s_{2}$ are given by (48) and (49), respectively.
Corollary 36. Under the assumptions of Theorem 34, if $\left|Y^{\prime}\right|$ is a uniform harmonic convex function, then

$$
\begin{aligned}
& \mid \frac{Y\left(b_{1}\right)+Y\left(b_{2}\right)}{2}+\frac{2 b_{1} b_{2}}{\alpha\left(b_{2}-b_{1}\right)}(Y \circ \Lambda)(k)-\frac{b_{1} b_{2} B(\alpha)}{\alpha\left(b_{2}-b_{1}\right)} \\
& \quad \times\left[\left(\begin{array}{l}
C F \\
1 / b_{2}
\end{array} I^{\alpha} Y \circ \Lambda\right)(k)+\left(\begin{array}{l}
C F \\
1 / b_{1}
\end{array} I^{\alpha} Y \circ \Lambda\right)(k)\right] \mid \\
& \leq \frac{b_{1} b_{2}\left(b_{2}-b_{1}\right)}{2} s_{15}^{1 / p}\left[s_{3}\left|Y^{\prime}\left(b_{1}\right)\right|^{q}+s_{4}\left|Y^{\prime}\left(b_{2}\right)\right|^{q}\right. \\
& \left.-s_{5} \psi\left(\left|\frac{1}{b_{2}}-\frac{1}{b_{1}}\right|\right)\right]^{1 / q},
\end{aligned}
$$

where $s_{3}, s_{4}$, and $s_{5}$ are given by (51), (52), and (53), respectively.

Corollary 37. Under the assumptions of Theorem 34, if $\left|Y^{\prime}\right|$ is a uniform harmonic s-convex function, then

$$
\begin{gathered}
\mid \frac{Y\left(b_{1}\right)+Y\left(b_{2}\right)}{2}+\frac{2 b_{1} b_{2}}{\alpha\left(b_{2}-b_{1}\right)}(Y \circ \Lambda)(k)-\frac{b_{1} b_{2} B(\alpha)}{\alpha\left(b_{2}-b_{1}\right)} \\
\quad \times\left[\left(\begin{array}{c}
C F \\
1 / b_{2}
\end{array} I^{\alpha} Y \circ \Lambda\right)(k)+\left({ }_{1 / b_{1}}^{C F} I^{\alpha} Y \circ \Lambda\right)(k)\right] \mid \\
\leq \frac{b_{1} b_{2}\left(b_{2}-b_{1}\right)}{2} s_{15}^{1 / p}\left[s_{6}\left|Y^{\prime}\left(b_{1}\right)\right|^{q}+s_{7}\left|Y^{\prime}\left(b_{2}\right)\right|^{q}\right. \\
\left.-s_{8} \psi\left(\left|\frac{1}{b_{2}}-\frac{1}{b_{1}}\right|\right)\right]^{1 / q},
\end{gathered}
$$

where $s_{6}, s_{7}$, and $s_{8}$ are given by (55), (56), and (57), respectively.

Theorem 38. If $Y:\left[b_{1}, b_{2}\right] \longrightarrow \mathbb{R}$ is a uniform harmonic $\chi$-convex function with $b_{1}<b_{2}$ and $i \in[0,1]$, then we have

$$
\begin{aligned}
& \mid \frac{Y\left(b_{1}\right)+Y\left(b_{2}\right)}{2}+\frac{2 b_{1} b_{2}}{\alpha\left(b_{2}-b_{1}\right)}(Y \circ \Lambda)(k)-\frac{b_{1} b_{2} B(\alpha)}{\alpha\left(b_{2}-b_{1}\right)} \\
& \times\left[\left(\begin{array}{l}
C F \\
1 / b_{2}
\end{array} I^{\alpha} Y \circ \Lambda\right)(k)+\left(\begin{array}{l}
C F \\
1 / b_{1}
\end{array} I^{\alpha} Y \circ \Lambda\right)(k)\right] \\
& \leq \frac{b_{1} b_{2}\left(b_{2}-b_{1}\right)}{2} s_{16}^{1 / p}\left[\int _ { 0 } ^ { 1 } | 1 - 2 i | ^ { p } \left(\chi(i)\left|Y^{\prime}\left(b_{1}\right)\right|^{q}\right.\right. \\
& \left.\left.+\chi(1-i)\left|Y^{\prime}\left(b_{2}\right)\right|^{q}-(1-i) \chi(i) \psi\left(\left|\frac{1}{b_{2}}-\frac{1}{b_{1}}\right|\right)\right) d i\right]^{1 / q}
\end{aligned}
$$

where

$$
s_{16}=\frac{b_{2}{ }^{1-2 p}-b_{1}^{1-2 p}}{\left(b_{2}-b_{1}\right)(1-2 p)} \text {. }
$$


Proof. Using Lemma 21, modulus property, and uniform harmonic $\chi$-convexity of $\left|Y^{\prime}\right|^{q}$, then we have

$$
\begin{aligned}
& \mid \frac{Y\left(b_{1}\right)+Y\left(b_{2}\right)}{2}+\frac{2 b_{1} b_{2}}{\alpha\left(b_{2}-b_{1}\right)}(Y \circ \Lambda)(k)-\frac{b_{1} b_{2} B(\alpha)}{\alpha\left(b_{2}-b_{1}\right)} \\
& \times\left[\left(\begin{array}{l}
C F \\
1 / b_{2}
\end{array} I^{\alpha} Y \circ \Lambda\right)(k)+\left({ }_{1 / b_{1}}^{C F} I^{\alpha} Y \circ \Lambda\right)(k)\right] \mid \\
& \leq \frac{b_{1} b_{2}\left(b_{2}-b_{1}\right)}{2} \int_{0}^{1}\left|\frac{1-2 i}{\left((1-i) b_{1}+i b_{2}\right)^{2}}\right| Y^{\prime}\left(\frac{b_{1} b_{2}}{(1-i) b_{1}+i b_{2}}\right) \mid d i \\
& \leq \frac{b_{1} b_{2}\left(b_{2}-b_{1}\right)}{2}\left(\int_{0}^{1}\left|\frac{1}{\left((1-i) b_{1}+i b_{2}\right)^{2}}\right|^{p}\right)^{1 / p} \\
& \times\left[\int _ { 0 } ^ { 1 } ( 1 - 2 i ) ^ { q } \left(\chi(i)\left|Y^{\prime}\left(b_{1}\right)\right|^{q}+\chi(1-i)\left|Y^{\prime}\left(b_{2}\right)\right|^{q}\right.\right. \\
& \left.\left.-(1-i) \chi(i) \psi\left(\left|\frac{1}{b_{2}}-\frac{1}{b_{1}}\right|\right)\right)\right]^{1 / q} d i \text {. }
\end{aligned}
$$

This completes the proof.

Now, we discuss some special cases of Theorem 38:

(i) If we take $\chi(i)=1$ in Theorem 38, then we have the result for uniform harmonic $P$-convex functions

(ii) If we take $\chi(i)=i$ in Theorem 34 and use a similar technique as in Corollary 39, then we have the result for uniform harmonic convex functions

Corollary 39. Under the assumptions of Theorem 38, if $\left|Y^{\prime}\right|$ is a uniform harmonic P-convex function, then

$$
\begin{aligned}
& \mid \frac{Y\left(b_{1}\right)+Y\left(b_{2}\right)}{2}+\frac{2 b_{1} b_{2}}{\alpha\left(b_{2}-b_{1}\right)}(Y \circ \Lambda)(k)-\frac{b_{1} b_{2} B(\alpha)}{\alpha\left(b_{2}-b_{1}\right)} \\
& \times\left[\left(\begin{array}{l}
C F \\
1 / b_{2}
\end{array} I^{\alpha} Y \circ \Lambda\right)(k)+\left(\begin{array}{l}
C F \\
1 / b_{1}
\end{array} I^{\alpha} Y \circ \Lambda\right)(k)\right] \mid \\
& \leq \frac{b_{1} b_{2}\left(b_{2}-b_{1}\right)}{2} s_{16}^{1 / p}\left[\int_{0}^{1 / 2}(1-2 i)^{p}\right. \\
& \times\left(\left|Y^{\prime}\left(b_{1}\right)\right|^{q}+\left|Y^{\prime}\left(b_{2}\right)\right|^{q}-(1-i) \psi\left(\left|\frac{1}{b_{2}}-\frac{1}{b_{1}}\right|\right)\right) d i \\
& +\int_{1 / 2}^{1}(2 i-1)^{p}\left(\left|Y^{\prime}\left(b_{1}\right)\right|^{q}+\left|Y^{\prime}\left(b_{2}\right)\right|^{q}\right. \\
& \left.\left.-(1-i) \psi\left(\left|\frac{1}{b_{2}}-\frac{1}{b_{1}}\right|\right)\right) d i\right]^{1 / q} \\
& \leq \frac{b_{1} b_{2}\left(b_{2}-b_{1}\right)}{2} s_{16}^{1 / p}\left[\frac{1}{q+1}\left[\left|Y^{\prime}\left(b_{1}\right)\right|^{q}+\left|Y^{\prime}\left(b_{2}\right)\right|^{q}\right]\right. \\
& \left.-\frac{1}{2(q+1)} \psi\left(\left|\frac{1}{b_{2}}-\frac{1}{b_{1}}\right|\right)\right]^{1 / q} \text {, }
\end{aligned}
$$

Corollary 40. Under the assumptions of Theorem 34, if $\left|Y^{\prime}\right|$ is a uniform harmonic convex function, then

$$
\begin{gathered}
\mid \frac{Y\left(b_{1}\right)+Y\left(b_{2}\right)}{2}+\frac{2 b_{1} b_{2}}{\alpha\left(b_{2}-b_{1}\right)}(Y \circ \Lambda)(k)-\frac{b_{1} b_{2} B(\alpha)}{\alpha\left(b_{2}-b_{1}\right)} \\
\quad \times\left[\left({ }_{1 / b_{2}}^{C F} I^{\alpha} Y \circ \Lambda\right)(k)+\left({ }_{1 / b_{1}}^{C F} I^{\alpha} Y \circ \Lambda\right)(k)\right] \mid \\
\leq \frac{b_{1} b_{2}\left(b_{2}-b_{1}\right)}{2} s_{16}^{1 / p}\left[\frac{\left|Y^{\prime}\left(b_{1}\right)\right|^{q}+\left|Y^{\prime}\left(b_{2}\right)\right|^{q}}{2(q+1)}\right. \\
\left.-\frac{1}{2(q+1)(q+3)} \psi\left(\left|\frac{1}{b_{2}}-\frac{1}{b_{1}}\right|\right)\right]^{1 / q},
\end{gathered}
$$

where $s_{16}$ is given by (74).

\section{Applications}

In this section, we present some applications to special means. We now recall some special means for two nonnegative numbers:

(1) The Arithmetic Mean. $A\left(b_{1}, b_{2}\right):=\left(b_{1}+b_{2}\right) / 2$

(2) The Geometric Mean. $G\left(b_{1}, b_{2}\right):=\sqrt{b_{1} b_{2}}$

(3) The Harmonic Mean. $H\left(b_{1}, b_{2}\right):=2 b_{1} b_{2} /\left(b_{1}+b_{2}\right)$

(4) The Logarithmic Mean. $L\left(b_{1}, b_{2}\right):=\left(b_{2}-b_{1}\right) /\left(\ln b_{2}-\ln b_{1}\right)$

(5) The $p$-Logarithmic Mean. $L_{p}\left(b_{1}, b_{2}\right):=\left(\left(b_{2}{ }^{p+1}-b_{1}{ }^{p+1}\right) /(p\right.$ $\left.+1)\left(b_{2}-b_{1}\right)\right)^{1 / p}, p \in \mathbb{R}-\{-1,0\}$

Proposition 41. For $b_{1}, b_{2}>0$, we have

$$
\begin{gathered}
\left|A\left(b_{1}{ }^{n+2}, b_{2}{ }^{n+2}\right)+\frac{\left(b_{1}+b_{2}\right)}{\left(b_{2}-b_{1}\right) k^{n+2}} H\left(b_{1}, b_{2}\right)-G^{2}\left(b_{1}, b_{2}\right) E_{n}^{n}\left(b_{1}, b_{2}\right)\right| \\
\leq \frac{G^{2}\left(b_{1}, b_{2}\right)\left(b_{2}-b_{1}\right)}{2}\left(\frac{1}{1+p}\right)^{1 / p} \\
\quad \times\left[(n+2)^{q} s_{9} b_{1}^{q(n+1)}+(n+2)^{q} s_{10} b_{2}^{q(n+1)}\right]^{1 / q},
\end{gathered}
$$

where $s_{9}$ and $s_{10}$ are given by (62) and (63), respectively.

Proof. For $\alpha=1, \psi=0$, and $Y(i)=i^{n+2}$ in Corollary 30, we obtain our required result.

Proposition 42. For $b_{1}, b_{2}>0$, we have

$$
\begin{aligned}
& \mid \frac{\mu}{\lambda+2 \mu} A\left(b_{1}{ }^{(\lambda / \mu)+2}, b_{2}{ }^{(\lambda / \mu)+2}\right)+\frac{(\lambda+2 \mu)\left(b_{1}+b_{2}\right)}{\mu\left(b_{2}-b_{1}\right) k^{(\lambda / \mu)+2}} H\left(b_{1}, b_{2}\right) \\
& \quad-\frac{(\lambda+2 \mu)}{\mu} G^{2}\left(b_{1}, b_{2}\right) E_{\lambda / \mu}^{\lambda / \mu}\left(b_{1}, b_{2}\right) \mid \\
& \quad \leq \frac{G^{2}\left(b_{1}, b_{2}\right)\left(b_{2}-b_{1}\right)}{2}\left(\frac{1}{1+p}\right)^{1 / p}\left[s_{9} b_{1}{ }^{(q \lambda+q \mu) / \mu}+s_{10} b_{2}{ }^{(q \lambda+q \mu) / \mu}\right]^{1 / q} .
\end{aligned}
$$

where $s_{16}$ is given by (74). 
Proof. For $\alpha=1, \psi=0$, and $Y(i)=(\mu /(\lambda+2 \mu)) i^{(\lambda / \mu)+2}$ in Corollary 30 , we obtain our required result.

Proposition 43. For $b_{1}, b_{2}>0$, we have

$$
\begin{gathered}
\left|A\left(b_{1}{ }^{n+2}, b_{2}{ }^{n+2}\right)+\frac{\left(b_{1}+b_{2}\right)}{\left(b_{2}-b_{1}\right) k^{n+2}} H\left(b_{1}, b_{2}\right)-G^{2}\left(b_{1}, b_{2}\right) E_{n}^{n}\left(b_{1}, b_{2}\right)\right| \\
\leq \frac{G^{2}\left(b_{1}, b_{2}\right)\left(b_{2}-b_{1}\right)}{2} s_{11}^{1 / p}\left[\frac{(n+2)^{q}}{q+1} A\left(b_{1}^{q(n+1)}, b_{2}^{q(n+1)}\right)\right]^{1 / q},
\end{gathered}
$$

where $s_{11}$ is given by (74).

Proof. For $\alpha=1, \psi=0$, and $Y(i)=i^{n+2}$ in Corollary 40, we obtain our required result.

Proposition 44. For $b_{1}, b_{2}>0$, we have

$$
\begin{aligned}
& \mid \frac{\mu}{\lambda+2 \mu} A\left(b_{1}{ }^{(\lambda / \mu)+2}, b_{2}{ }^{(\lambda / \mu)+2}\right)+\frac{(\lambda+2 \mu)\left(b_{1}+b_{2}\right)}{\mu\left(b_{2}-b_{1}\right) k^{(\lambda / \mu)+2} H\left(b_{1}, b_{2}\right)} \\
& \quad-\frac{(\lambda+2 \mu)}{\mu} G^{2}\left(b_{1}, b_{2}\right) E_{\lambda / \mu}^{\lambda / \mu}\left(b_{1}, b_{2}\right) \mid \\
& \leq \frac{G^{2}\left(b_{1}, b_{2}\right)\left(b_{2}-b_{1}\right)}{2} s_{11}^{1 / p}\left[\frac{A\left(b_{1}^{(q \lambda+q \mu) / \mu}, b_{2}{ }^{(q \lambda+q \mu) / \mu}\right)}{q+1}\right]^{1 / q} .
\end{aligned}
$$

Proof. For $\alpha=1, \psi=0$, and $Y(i)=(\mu /(\lambda+2 \mu)) i^{(\lambda / \mu)+2}$ in Corollary 40 , we obtain our required result.

\section{Data Availability}

No data were used to support this study.

\section{Conflicts of Interest}

The authors declare that they have no competing interests.

\section{Authors' Contributions}

All authors contributed equally to the writing of this paper. All authors read and approved the final manuscript.

\section{Acknowledgments}

This research was funded by Dirección de Investigación from Pontificia Universidad Católica del Ecuador in the research project entitled "Some integrals inequalities and generalized convexity" (Algunas desigualdades integrales para funciones con algún tipo de convexidad generalizada y aplicaciones).

\section{References}

[1] S. S. Dragomir and C. E. M. Pearce, Selected Topics on HermiteHadamard Inequality and Applications, Victoria University, Melbourne, Australia, 2000.

[2] I. Işcan, "Hermite-Hadamard type inequalities for harmonically convex functions," Hacettepe Journal of Mathematics and Statistics, vol. 6, no. 1014, pp. 935-942, 2014.

[3] M. Z. Sarikaya, E. Set, H. Yaldiz, and N. Basak, "Hermite-Hadamard's inequalities for fractional integrals and related fractional inequalities," Mathematical and Computer Modelling, vol. 57, no. 9-10, pp. 2403-2407, 2013.

[4] I. Işcan and S. Wu, "Hermite-Hadamard type inequalities for harmonically convex functions via fractional integrals," Applied Mathematics and Computation, vol. 238, pp. 237-244, 2014.

[5] M. U. Awan, M. A. Noor, M. V. Mihai, and K. I. Noor, "Inequalities via harmonic convex functions: conformable fractional calculus approach," Journal of Mathematical Inequalities, vol. 12, no. 1, pp. 143-153, 2018.

[6] M. A. Noor, K. I. Noor, M. U. Awan, and S. Costache, "Some integral inequalities for harmonically $h$-convex functions," UPB Scientific Bulletin, Series A: Applied Mathematics and Physics, vol. 77, no. 1, pp. 5-16, 2015.

[7] H. H. Bauschke and P. L. Combettes, Convex Analysis and Monotone Operator Theory in Hilbert Spaces, Springer Verlag, 2011.

[8] T. Abdeljawad and D. Baleanu, "On fractional derivatives with exponential kernel and their discrete versions," Reports on Mathematical Physics, vol. 80, no. 1, pp. 11-27, 2017.

[9] M. Caputo and M. Fabrizio, "A new definition of fractional derivative without singular kernal," Progress in Fractional Differentiation and Applications, vol. 1, no. 2, pp. 73-85, 2015. 